\title{
Ein (un)deutsches Juristenleben - Franz Neumann zum 80. Geburtstag
}

Am 23. Mai I 980 wäre Franz Neumann 80 Jahre alt geworden. Wenn diesem 1954 tödlich verunglückten deutsch-amerikanischen Juristen und Politikwissenschaftler erst in allerjüngster Zeit die Publizität zuteil wird, die seinem praktischen und theoretischen Werk gebührt', so ist dies kein Zufall. Es spiegelt sich darin ein Versäumnis, das das Verhältnis der Deutschen zu ihrer Geschichte ein weiteres Mal als Verdrängungsprozeß erscheinen läßt. Juristen spielten, mehr als andere Vertreter der Akademikerschicht, eine unrühmliche Rolle bei der Reetablierung des autoritären Staates in der Weimarer Republik². Die Biographie Franz Neumanns kann als exemplarisch für die Möglichkeit des Gegenteils gelten - und für die Voraussetzungen und Folgen davon. ${ }^{3}$ Entschiedener Gegner des Nationalsozialismus, der er war, hat er bis zuletzt, im Gegensatz zu den vielen wirklich Kompromittierten, die Schuldfrage in fast selbstquälerisch zu nennender Weise an sich selber gerichtet. "Warum ich das Land so liebe und doch so verabscheue? «, schreibt er noch 1954 in einem Brief an Helge Pross, und er gibt als Antwort: „Vielleicht ist es ein Schuldgefühl, das ganz tief sitzt: Wie oft habe ich mir nach 1933 die Frage vorgelegt, wo meine Verantwortung für den Nationalsozialismus eigentlich steckt. Denn ich glaube an kollektive Schuld aber dann kann ich mich ja davon nicht ausnehmen . . . Wir, die wir in Opposition zu der Reaktion standen, waren alle zu feige. Wir haben alle kompromittiert. Ich habe ja mit eigenen Augen gesehen, wie verlogen die $\mathrm{SpD}$ in den Monaten Juli 1932 bis Mai r 933 war (und nicht nur damals) und habe nichts gesagt. Wie feige die Gewerkschaftsbosse waren - und ich habe ihnen weiter gedient. Wie verlogen die Intellektuellen waren - und ich habe geschwiegen. Natürlich kann ich das rational rechtfertigen mit der Einheitsfront gegen den Nationalsozialismus, aber im Grunde war Angst vor der Isolierung dabei . . . So habe ich mitgemacht bei dem Ausverkauf der Ideen der sogenannten deutschen Linken. Sicherlich ist mein Beitrag gering und der Politiker wird meine Haltung ironisch betrachten. Aber kann man den Verfall der SPD und den Aufstieg des Nationalsozialismus nur als politisches Problem betrachten? Waren da nicht moralische Entscheidungen zu treffen? Die habe ich zu spät und immer noch nicht radikal genug getroffen. « ${ }^{3 a}$

Mangel an wirklichen Perspektiven - dieses Signum der gegenwärtigen deutschen Rechtspolitik ist vielleicht auch die Folge mangelnden Gedächtnisses. Es bedarf

I Franz Neumann, Behemoth. Struktur und Praxis des Nationalsozialismus 1933-1944, Frankfurt/M. 1977; ders., Wirtschaft, Staat, Demokratie. Aufsätze 1930-1954, Frankfurt/M. 1978; ders., Die Herrschaft des Gesetzes, Frankfurt/M. 1980. Vorher war nur erschienen: ders., Demokratischer und autoritärer Staat, Frankfurt/M. 1967.

2 Heinrich Hannover / Elisabeth Hannover-Drück, Politische Justiz 1918-1933, Neuauflage Hamburg 1977 .

3 Vgl. ausführlicher meine Skizzen zu einer politischen und intellektuellen Biographie, in: Neumann, Wirtschaft ..., a. a. O. S. $7 \mathrm{ff}$.

3a Demokratischer und autoritärer Staat, a.a.O., S. I 2. 

rettenden Kritik, also der Erinnerung 4 .

\section{Arbeitsrecht und Wirtschaftsdemokratie - der juristische Praktiker (1918-1933)}

Franz Neumann, Sohn eines jüdischen Kaufmanns aus Kattowicz, studierte ab 1918 nach kurzem Kriegsdienst Jurisprudenz in Breslau, Rostock und vor allem in Frankfurt. Die Berührung mit der rätedemokratischen Bewegung in Leipzig, wo er aktiv am Barrikadenkampf der revolutionären Arbeiter und Soldaten teilnahm, lenkte sein Interesse frühzeitig auf den geschichtsmächtigen Konflikt zwischen Kapital und Arbeit. In der Auseinandersetzung zwischen sozialdemokratischer und kommunistischer Strategien, die die frühe Weimarer Republik bestimmte, schlug er sich alsbald auf die reformistische Seite. Damit sind die historischen und politischen Voraussetzungen genannt, die für einen Juristen der Weimarer Epoche Arbeitsrecht und Wirtschaftsdemokratie als die primären Arbeitsgebiete in den Vordergrund treten lassen mußten. Die Verbindung von juristischer Technik und Reformpolitik hinderte Neumann jedoch, anders als häufig in der Gegenwart, keineswegs an der Ausbildung genuin theoretischer Interessen. Im Gegenteil: Seine 1923 bei dem neukantianischen Rechtsphilosophen Max Ernst Mayer abgeschlossene Dissertation' unternahm den theoretisch ambitionierten Versuch, die Kluft von Sein und Sollen rechtssoziologisch zu schließen. Prägend für Neumanns politischen und intellektuellen Charakter wurden die Jahre zwischen 1923 und 1927 , in denen er, neben Referendarausbildung und Lehrtätigkeit an der Frankfurter Akademie der Arbeit, als persönlicher Assistent Hugo Sinzheimers arbeitete. In der Person dieses geistigen Vaters des Weimarer Arbeitsrechts wurde ihm die prekäre Stellung der jüdisch-sozialistischen Intelligenz im akademischen Leben von Weimar zur Erfahrung, schon bevor der nationalsozialistische Terror offen hervortrat. Neumann berichtet, daß er zu Anfang der 2oer Jahre den soeben ernannten Professor des Arbeitsrechts gegen antisemitische Angriffe von Seiten nationalistischer Studenten verteidigen helfen mußte ${ }^{6}$. Daß ein jüdischsozialistischer Intellektueller gleichwohl zunächst nicht notwendig "ortlos" sein mußte, beweist die Biographie Neumanns, stand er doch in der sozialistischen Bewegung eher auf der realistischen als auf der utopischen Seite. Die Stabilisierung der Verhältnisse ab 1924 konnte die reformistische Strategie, die auf Sozialpolitik und Verrechtlichung des Klassenkonflikts baute, als problemlose Vermittlung von Idee und Wirklichkeit erscheinen lassen.

Erste Selbständigkeit erlangte Neumann dann in Berlin ab 1928, wo er als Syndikus der Baugewerkschaft mit Lirnst Fraenkel, ebenfalls Schüler Sinzheimers, ein Anwaltsbüro teilte. Die explosive Produktivität, die er in dieser Funktion und verschiedenen Nebentätigkeiten (als Dozent für Arbeitsrecht an der neugegründeten Hochschule für Politik, als Mitarbeiter am 1927 eingerichteten Reichsarbeitsgericht in Leipzig) bei der Konstruktion und Konkretisierung des Arbeits- und Wirtschaftsrechts an den Tag legte, ist in einer Fülle von rechtstheoretischen Arbeiten dokumentiert.' In ihnen äußerte er sich nicht nur zu technischen Rechtsproblemen im engeren Sinn, vielmehr war deren Ausarbeitung in eine umfassende politische Strategie eingebettet, die die

\footnotetext{
4 Jürgen Habermas, Bewußtmachende oder rettende Kritik, in: ders., Kultur und Kritik, Frankfurt/M. 1973 , S. $302 \mathrm{ff}$.

s Rechtsphilosophische Einleitung zu einer Abhandlung über Staat und Strafe, Maschinenskript, Frankfurt/ M. 1923 .

6 Intellektuelle Emigration und Sozialwissenschaft, in: Neumann, Wirtschaft ..., a. a. O., S. 413.

7 Vgl. die Bibliographie von Wolfgang Luthardt, in: Neumann, Wirtschaft ..., a. a. O., S. ${ }_{4}^{62-464}$.
} 
Gewerkschaften auf der einen, den Staat auf der anderen Seite zum Ziel hatte. Diese

Strategie war insofern reformistisch, als sie den Sozialismus aus der immanenten Transformation des Kapitalismus, und zwar mit rechtspolitischen Mitteln, zu entwickeln glaubte. Sie hatte ihre eigene Dynamik, aber auch ihre Grenzen. So ließ Neumann, obschon weniger skeptisch eingestellt als Otto Kahn-Freund ${ }^{8}$, keinen Zweifel daran, daß die von Betriebsgemeinschaftsvorstellungen geprägte Rechtsprechung des Reichsarbeitsgerichts, einmal institutionalisiert, keineswegs den ursprünglichen Erwartungen der Arbeiterschaft gerecht wurde. Darin zeigte sich für ihn die Ambivalenz, die mit der Verrechtlichung von Klassenkämpfen, und d.h. der Delegation der Konfliktfälle an eine alles andere als sozialistisch eingestellte Richterschaft, gegeben war'. Wenn Neumann, wie seine Leidenschaft zur analytischen Unterscheidung belegt, von Anfang an Jurist mit Leib und Seele war, so lehrten ihn die Erfahrungen der Weimarer Zeit nicht minder vehement das Fürchten vor einer rein immanent-technisch verstandenen Ausübung dieses intellektuellen Handwerks. Gerade die intensive Beschäftigung mit dem neu entstandenen Arbeitsrecht schärften ihm mit der Hochschätzung der formalen Rechtsrationalität gleichzeitig den Sinn für die Möglichkeit ihres Mißbrauchs ein, für eine Möglichkeit, die im modernen Monopolkapitalismus ohnehin, unter Bedingungen einer geschwächten parlamentarischen Legislative zusätzlich wirksam schien. Als Promotoren des Verfalls der Rechtsrationalität diagnostizierte Neumann die in der Weimarer Zeit verstärkt vorgetragenen Forderungen der Freirechtsbewegung und die Versuche zur Einrichtung eines obersten Gerichts zur Uberprüfung der Verfassungsmäßigkeit von Gesetzen $^{10}$. Neumann erkannte sehr klar den politischen und sozialen Sinn, der in solchen Veränderungen der juristischen Theorie und Praxis gelegen war: die Entbindung der sozial reaktionären Richterschaft von Gesetzen, die die rechtliche Gleichheit zu einer sozialen Gleichheit fortzuentwickeln versuchten, und damit die Suspendierung des noch für den liberalen Rechtsstaat unbezweifelten Primats der Legislative gegenüber der Jidikative.

Nicht nur Neumanns Praxis, sondern auch seine Theorie ist bereits in der Weimarer Epoche deutlich durch außerjuristische, politische und soziologische Gedanken bestimmt. Er folgt hier dem Vorbild Hugo Sinzheimers und des Austromarxisten Karl Renner ${ }^{\mathrm{r}}$. Gleichwohl erkennt er, anders als etwa Otto Kirchheimer in seinen verfassungssoziologischen Studien ${ }^{12}$ auch nach der Weltwirtschaftskrise nicht den systematischen Charakter, den die Erosion des Weimarer Staats- und Rechtsgefüges seit Brünings Regierungsantritt hatte. Dies kommt deutlich zum Ausdruck in der Tatsache, daß sich Neumann ab 1929 statt auf die Krise auf Fragen der Wirtschaftsdemokratie konzentrierte - zu einem Zeitpunkt also, da die sozialen und politischen Grundlagen des Gewerkschaftsprogrammes von 1928 bereits zerstört waren; und es kommt zum Ausdruck in der Form, in der Neumann dies tut: seine Arbeiten zur Wirtschaftsdemokratie kaprizieren sich auf die verfassungs- und staatsrechtliche Dogmatik einer Demokratisierung der Wirtschaft, in der die Gewerkschaften

8 Das soziale Ideal des Reichsarbeitsgerichts (1931), in: Hrsg. Thilo Ramm, Arbeitsrecht und Politik, Neuwied 1966, S. I49 ff.

9 Die politische und soziale Bedeutung der arbeitsgerichtlichen Rechtssprechung (1929), in: Ramm, a. a. O., S. I 3 ff., wo Neumann sich u. a. auf Ernst Fraenkel, Zur Soziologie der Klassenjustiz, Berlin 1927 , beruft.

ro Richterliches Ermessen und Methodenstreit im Arbeitsrecht, in: Arbeitsrecht, Jg. 1929, Sp. 32 I ff.; Gegen ein Gesetz zur Nachprüfung der Verfassungsmäßigkeit von Reichsgesetzen, in: Die Gesellschaft, Jg. I929, S. $517 \mathrm{ff}$.

I1 Hugo Sinzheimer, Grundzüge des Arbeitsrechts, 2. Auflage, Jena 1927; Karl Renner, Die Rechtsinstitute des Privatrechts und ihre soziale Funktion, 2. Auflage, Tübingen 1929.

12 Z. B. Weimar - und was dann? (1930), in: Kirchheimer, Politik und Verfassung, Frankfurt/M. 1964, S. $9 \mathrm{ff}$. 
paritätisch mit den Unternehmern die entscheidende organisatorische Rolle spielen sollen ${ }^{\text {is. }}$.

Bei Neumanns verfassungstheoretischen Schriften aus der Zeit von 1930 bis 1933 ist die spezifische Situation zu beachten, über die wir heute mehr wissen. Neumann hat, wie die meisten seiner Zeitgenossen, nicht erkannt, daß schon mit der Regierung Brüning eine Arbeiterbewegung, die sich der Legalität einer durch Verfassungsdurchbrechung ausgehöhlten Verfassung unterwarf, zum Scheitern verurteilt war; denn auch Brüning, den die Sozialdemokraten als Schild gegen Hitler zu stemmen glaubten, setzte bewußt die Weimarer Reichsverfassung außer Kraft, ohne sie zu verändern. So illusionär Neumanns Ansatz unter diesen Bedingungen jedoch auch gewesen sein mag - nach 1945 hätten es die Sozialdemokraten und Gewerkschaften nicht versäumen dürfen, ihn bei der Schaffung des Grundgesetzes und der Festlegung der zukünftigen Verfassungs- und Rechtspolitik zur Geltung zu bringen!

Versucht man eine zusammenfassende Charakterisierung des Typus von Juristen, zu dem Neumann mit Ernst Fraenkel, Otto Kahn-Freund und andern zu zählen ist, so ist auf die komplexe Verflechtung von sozialrechtlichen, politisch-staatlichen und sozialwissenschaftlichen Tendenzen hinzuweisen. Wenn er sich aus der Perspektive der traditionellen Rechtswissenschaft als Randfigur darstellt, so gereicht ihm dies aus größerer historischer Distanz gerade zu Ehre. Der juristische Außenseiter-Status war es nämlich, der ihn immun machte gegen den profaschistischen Bazillus, als der sich die Uberwindung des liberalen Rechtsformalismus alsbald erweisen sollte. Dabei stellt sich ein Paradox heraus: Verglichen mit der Rematerialisierung der Rechtsrationalität, die ein Carl Schmitt und seine antidemokratischen Anhänger betrieben, erweisen sich die Juristen der Arbeiterbewegung als traditionell - als Rechtspositivisten. Sie nahmen den Formalismus des bürgerlichen Rechtssystems ernster als seine bürgerlichen Erneuerer ${ }^{14}$. In politischer Hinsicht erweisen sie sich freilich auch als legalistisch, versteht man darunter nicht nur die Treue zum geschriebenen Recht, sondern ein übergroßes Vertrauen in die Unverbrüchlichkeit einer Rechtsordnung, die in der Wirtschaftskrise keinen faktischen Garanten mehr hinter sich hatte's. In dieser Hinsicht teilten Neumann und seine Kollegen das Schicksal, das den Reformismus am Ende der Weimarer Republik generell ereilte, ein Schicksal, das, wie Neumann wenig später selbstkritisch anmerkt, »sowohl unvermeidlich wie selbstverschuldet« $\operatorname{war}^{16}$.

\section{Vom Liberalismus zum Faschismus - der Rechtstheoretiker (1933-1942)}

Franz Neumann, der noch 1932 zum Syndikus der SPD berufen worden war und in dieser Funktion gegen die Beschränkungen der Pressefreiheit kämpfte ${ }^{17}$, mußte Anfang Mai 1933, nach der Besetzung der Gewerkschaftshäuser durch die SA, Deutschland bei Nacht und Nebel verlassen. Damit war die politische Logik eines kritischen Juristenlebens der Weimarer Republik vollstreckt. Die erste Station der Emigration war London, wo Neumann durch die Vermittlung des sozialistischen Politikwissenschaftlers Laski ein Stipendium der London School of Economics

${ }_{3}$ Die soziale Bedeutung der Grundrechte in der Weimarer Verfassung (1930), in: Neumann, Wirtschaft ..., a. a. O., S. 57 ff.; ders., Koalitionsfreiheit und Reichsverfassung, Berlin 1932.

14 Ingeborg Maus, Bürgerliche Rechtstheorie und Faschismus, München 1976.

is Wolfgang Luthardt, Sozialdemokratie und Legalstrategie, in: Geschichte als politische Wissenschaft, Stuttgart 1979, S. $142 \mathrm{ff}$.

I6 Der Niedergang der deutschen Demokratie (1933), in: Neumann, Wirtschaft ..., a. a. O., S. 104.

17 Die öffentlichrechtlichen und privatrechtlichen Beschränkungen der Pressefreiheit, Berlin 1932. 
erhielt. War die Aufmerksamkeit zuerst noch unmittelbar auf die Entwicklung in Deutschland gerichtet, was Beobachtung der nationalsozialistischen Politik, Selbstkritik des Reformismus und Unterstützung des inneren Widerstandes hieß, ${ }^{18}$ so besann sich der ehemalige juristische Praktiker rasch auf die genuinen Möglichkeiten, die ihm die Emigration noch beließ. Sie verwiesen ihn, nachdem er die Nutzlosigkeit der kontinentaleuropäischen Jurisprudenz im englischen case law erkannt hatte, auf das Gebiet der Theoriebildung und Forschung.

Es ist für die Geschichte der deutschen Rechtswissenschaft nach 1933 symptomatisch, daß ihre kritische Fortentwicklung extern stattfinden mußte und daß sie einen Weg aus der juristischen Immanenz heraus und hin zur politischen und soziologischen Thematisierung des Rechts nahm. Die London School of Economics, an der ein interdisziplinärer und komparativer Ansatz vorherrschte, erwies sich als ein idealer Ort um diesen Prozeß zu beschleunigen. Hier wandelte sich Neumann, unter der Anleitung durch Laski und Mannheim, vom Rechts- zum Politik- und Sozialwissenschaftler, wobei ihm der Gegenstand des Rechts nicht etwa verloren ging, sondern zum Nukleus wurde, in dem er die entscheidenden historischen Entwicklungslinien der neueren Geschichte zusammenzuziehen versuchte. Seine 1936 abgeschlossene, soeben auf Deutsch erschienene Arbeit "The Governance of the Rule of Law « legt davon Zeugnis $\mathrm{ab}^{19}$. Sie ist, worauf Neumann selber hinweist ${ }^{20}$, die theoretisch angeleitete Extrapolation der Erfahrung von Weimar auf die Rechtsgeschichte der bürgerlichen Gesellschaft vom Liberalismus zum Faschismus. Vermittelt über die als vorbildlich gewertete Studie Karl Renners und die wissenssoziologische Methode Karl Mannheims, stellt sich Neumann unter den Anspruch von zwei Theoretikern, die in Deutschland zwar bereits zu den sozialwissenschaftlichen Klassikern zählten, jedoch auf die allerneueste Rechtsentwicklung kaum Anwendung gefunden hatten: Karl Marx und Max Weber. Von ihnen übernimmt er das Mißtrauen gegen die methodische Isolierung der Rechtsideen von den Rechtspraktiken, von ihnen übernimmt er auch die Suche nach den gesellschaftlichen Strukturzusammenhängen, auf die hin beides: juristische Theorie und Praxis zu verorten sind. Die so zustande kommende, historisch und theoretisch gleichermaßen ambitionierte Konzeption ist Franz Neumanns rechtstheoretisches Vermächtnis ${ }^{21}$.

Der Typus von Rechtswissenschaft, den Neumann in den zoer Jahren ausarbeitete, läßt sich methodologisch charakterisieren nach dem Verhältnis, in das Rechtsdogmatik, Rechtsphilosophie und Rechtssoziologie eintreten. In Wendung gegen die Reine Rechtslehre von Hans Kelsen einerseits, gegen die Materialisierung des Rechts in der ontologisierten Souveränitätslehre von Carl Schmitt andererseits kommt Neumann zu einer Position, die der normativen Jurisprudenz ihr Recht beläßt, der Rechtssoziologie jedoch die übergreifende Aufgabe, auch gegenüber der Rechtsphilosophie, zuweist ${ }^{22}$. Ihr systematisches Zentrum ist einmal, im Anschluß an Max Weber, die soziologische Lehre von der Staatssouveränität, zum andern eine juristisch formulierte, jedoch historisch und soziologisch informierte Typologie der Grundrechte. Diese methodologische Dimensionierung der Rechtswissenschaft, in der sich Neumanns neukantianischer Ausgangspunkt wiederholt, kommt einmal zur Anwendung in einer umfangreich angelegten Ideen- und Theoriegeschichte des bürgerlichen Rechtsstaates, die sich an dem Widerspruch von Freiheitsrechten und

18 Z. B. The State and Labour in Germany, in: The Contemporary Review 1934, S. 713 ff.; Rechtsstaat, Gewaltenteilung und Sozialismus (5934); Die Gewerkschaften in Demokratie und Diktatur (1935), beide in: Neumann, Wirtschaft ..., a. a. O., S. 124 ff., S. 145 ff.

19 Die Herrschaft des Gesetzes, a. a. O.

20 Ebenda, S. I9.

2I Vgl. mein wissenschaftsgeschichtliches und wissenschaftspolitisches Nachwort, ebenda, S. $357 \mathrm{ff}$.

22 Ebenda, Teil I, S. 27 ff. 
Souveränität abarbeitet - mit dem Ergebnis einer konsequenten Positivierung des Naturrechts ${ }^{23}$; zum andern führt sie zur Konstruktion eines Idealtyps des bürgerlichen Gesetzgebungsstaates, dessen vergleichsweise reinste historische Realisierung Neumann im klassischen Parlamentarismus des englischen Liberalismus gegeben sieht. Die gleichermaßen juristische und soziologische Ausarbeitung dieses Idealtyps geschieht auf einer funktionalen und einer systematischen Ebene. Systematisch gesehen differenziert sich in der bürgerlichen Gesellschaft ein Rechtssystem aus, dessen prekäre halbautonomer Charakter am Status der Grundrechte einerseits, an den dominanten Eigenschaften des Gesetzes (Allgemeinheit, Bestimmtheit, Nichtrückwirkung, Gesetzesbindung des Richters) festgemacht ist. Die funktionale Verortung dieses Rechtssystems geschieht auf dreifacher Weise: ökonomisch kommt ihm die Funktion zu, die ökonomischen Prozesse berechenbar zu machen; politisch kommt ihm die Funktion zu, die Vorherrschaft des Bürgertums gleichzeitig zu sichern und zu verdecken; kulturell kommt ihm die Funktion zu, dem Individuum ein Minimum an Rechtssicherheit und Gerechtigkeit zu garantieren. Dieser Idealtypus spielte in den historischen Forschungen, die Neumann in den zoer Jahren durchführte, eine methodologische Doppelrolle: er war forschungstechnischer wie normativer Bezugspunkt, theoretischer wie idealer Ausdruck der Rationalität, die in der bürgerlichen Konkurrenzgesellschaft gleichermaßen angelegt und verunmöglicht ist. Der deutsche Rechtsstaat und der englische Parlamentarismus erschienen dabei als zwei verschiedene Varianten dieser Rationalität. ${ }^{24}$

Die Entwicklung, die Neumann im Londoner Exil vom reformistischen Rechtspraktiker zum materialistischen Rechtstheoretiker machte, ist nicht bloß rechtstheoretisch von Bedeutung. Sie zeichnete die Perspektiven vor, in deren Verfolgung die Rechtswissenschaft in einem ausgezeichneten Sinn zum Zentrum einer Theorie der bürgerlichen Gesellschaft zu werden vermag. Hier liegt auch der wissenschaftsinterne Grund, der es Neumann 1937 ermöglichte, zum Mitarbeiter am emigrierten Institut für Sozialforschung Max Horkheimers zu werden. ${ }^{25}$ Wenn hier, wie die wissenschaftsgeschichtliche Forschung heute belegt ${ }^{26}$, die marxistische Theoriebildung ihren fortgeschrittensten Stand, das Stadium einer disziplinär ausdifferenzierten und interdisziplinär rückgekoppelten Sozialforschung erreichte, so spielte Neumann, zusammen mit Otto Kirchheimer, den rechts- und staatstheoretischen Part im kongenialen Forschungsteam ${ }^{27}$. An ihren Arbeiten in der Zeitschrift für Sozialforschung läßt sich, mehr als an den neueren marxistischen Staats- und Rechtsableitungen, ablesen, daß eine akzeptable materialistische Rechtswissenschaft nur in engem Kontakt mit weit auseinander liegendem rechtsgeschichtlichen Material entwickelt werden kann. Dieses Material, das der deutschen, englischen und französischen Rechtsgeschichte entsprang, organisierte Neumann zu einer großangelegten, Liberalismus, Massendemokratie und Faschismus umfassenden These, derzufolge die formale Rationalität des Rechts unter dem Druck der ökonomischen und politischen Monopolbildung transformiert, rematerialisiert und letztlich zerstört wird. Als Indikatoren dieses Verfallsprozesses nannte er u. a. die Generalklausel-Jurisprudenz, die Freirechtsbewegung, die Maßnahmegesetzgebung und generell das Wiederaufleben des Naturrechts ${ }^{28}$.

23 Ebenda, Teil II, S. $77 \mathrm{ff}$

24 Ebenda, Teil III, S. $199 \mathrm{ff}$

2) Neumann leistete sein Debut in der Zeitschrift für Sozialforschung von 1937 mit dem Aufsatz: Der Funktionswandel des Gesetzes im Recht der bürgerlichen Gesellschaft, in: Neumann, Demokratischer und autoritärer Staat, a. a. O., S. $28 \mathrm{ff}$.

26 Helmut Dubiel, Wissenschaftsorganisation und politische Erfahrung, Frankfurt/M. 1978, S. 135 ff.

27 Vgl. mein Geschichte und Herrschaft, Frankfurt/M. 1979, S. 86 ff.

28 Die Herrschaft des Gesetzes, a. a. O., S. $314 \mathrm{ff}$. 
Die hauptsächliche Anstrengung jedoch, die Neumann zwischen 1937 und 1942 unternahm, bezog sich nicht mehr auf Probleme der Rechtstheorie und Rechtsforschung im engeren Sinn, sondern auf den deutschen Nationalsozialismus im Ganzen. Der 1942 zuerst publizierte "Behemoth «, der ihm alsbald internationales Renomee verschaffte, ist eine umfassende Analyse der politischen, ökonomischen, sozialen und ideologischen Struktur Hitler-Deutschlands. Auf diese Arbeit, die eine der wenigen theoretisch und empirisch gleichermaßen durchgeführten Gesamtdarstellungen einer modernen Gesellschaftsordnung darstellt, ist hier nicht einzugehen; hingewiesen sei nur auf den darin vorgenommenen Paradigmenwechsel von einer staatsrechtlichen zu einer soziologischen Herrschaftsanalyse, auf die These von der Dominanz der monopolkapitalistischen über die plankapitalistischen Elemente der Ókonomie, die eine bestimmte Reformulierung der Marxschen Kritik der Politischen Ókonomie impliziert, und auf die Darstellung der Sozialstruktur als Formation anarchisch konkurrierender Herrschaftseliten. Die neuere historische Detailforschung belegt heute die fortgesetzte Adäquanz des von Neumann ausgearbeiteten Bezugsrahmen ${ }^{29}$. Für den engeren Bereich des Rechts kam Neumann zu dem Resultat, daß der Nationalsozialismus mit äußerster Konsequenz an den Tag brachte, worauf die historische Logik der Krise von Weimar gezielt hatte: auf die Zerstörung der demokratischen Errungenschaften und auf die Reintegration der Arbeiterklasse in den imperialistischen Kapitalismus. Dementsprechend sah er die gravierendsten Rechtsveränderungen auf den Gebieten des Arbeitsrechts, des politischen Strafrechts und der demokratischen und individuellen Freiheitsrechte gegeben. Sie liefen auf die Substitution der Rechtsrationalität durch Terror hinaus, der sich hinter der völkischen Naturrechtsideologie zu verbergen suchte. Verglichen damit konnte das bürgerliche Recht, das immerhin Klassenrecht war, wie eine in die Ferne gerückte Idylle an rechtlich vermittelter Rationalität erscheinen. ${ }^{30}$

\section{Demokratie und Freibeit - der Politikwissenschaftler (1942-1954)}

I942 verließ Franz Neumann das Institut für Sozialforschung und trat, zusammen mit Kirchheimer, Herbert Marcuse und Arcadius Gurland, in die Dienste des amerikanischen State Department. Da der Nationalsozialismus nicht von innen heraus überwindbar schien, lag die Möglichkeit für einen effektiven antifaschistischen Kampf nur mehr im Bündnis mit der Macht, die als einzige vom Nationalsozialismus unbehelligt geblieben war. Im Office of Strategic Services (OSS), wo er ab I945 als Leiter der Deutschlandabteilung der Research and Analysis Branch tätig war, erstellte Neumann umfangreiche Studien über verschiedene Probleme wie ökonomische Kriegsverbrechen und Entnazifizierung, über die Funktion der Militärregierung und den Charakter des künftigen deutschen Staates, über die sozialen Kräfteverhältnisse nach dem Kriege usw. ${ }^{3 r}$ In der praktischen Politikberatung liegen Ursprung und Richtung der Entwicklung begründet, die Neumann in seiner dritten, der letzten Schaffensperiode genommen hat. Wiederum zeichnen sich an dieser Biographie mehr die Abwege als die Wege der deutschen Geschichte ab - sowohl was die neuerliche Verwandlung des Rechtstheoretikers zum Politikwissenschaftler wie was den politischen Bezugspunkt dieser Verwandlung betrifft.

29 Gert Schäfer, Franz Neumanns Behemoth und die heutige Faschismusdiskussion, in: Behemoth, a. a. O., S. $663 \mathrm{ff}$.

30 ebenda, besonders S. $68 \mathrm{ff}$., $307 \mathrm{ff} ., 478 \mathrm{ff}$., $509 \mathrm{ff}$.

31 Diese Arbeiten erscheinen 1981 bei der EVA unter dem Titel: Demokratischer Wiederaufbau Deutschlands aus der Emigration. 
Sämtliche seiner nach 1947 publizierten Arbeiten kreisen um die Sorge, wie in Deutschland von neuem eine stabile Demokratie aufgebaut werden könne.32 Charakteristisch für seine Deutschland-Analysen ist eine Akzentsetzung, die sich wohltuend vom Moralismus der "Stunde Null« unterscheidet: So sehr Neumann die nationalpsychologischen Wurzeln des Nationalsozialismus betonte, so wenig ließ er sich darüber hinwegtäuschen, was die strukturellen, also die ökonomischen, sozialen und politischen Gründe für die Entstehung des deutschen Ungeistes waren - und diese waren, angesichts der innerdeutschen wie der internationalen Konstellation, auf dem besten Weg zu überdauern. Neumann vertrat die auch bei den Amerikanern unpopuläre These, daß die Demokratie in Deutschland ohne grundlegende Veränderung der monopolkapitalistischen Wirtschaft, ohne Entlassung der reaktionären Beamten- und Richterschaft, also Maßnahmen, die über Entmilitarisierung und die ohnehin bald obsolete Entnazifizierung hinausgingen, kaum eine Chance habe ${ }^{33}$. Vor allem warnte er, seine justizkritische Vergangenheit aufgreifend, vor dem, was er den "Verfassungsfetischismus« der Grundgesetzväter nannte ${ }^{34}$. Neben der generellen Illusion über die Verrechtlichung von Konfliktlagen, der schon der Weimarer Reformismus verfallen war, verstand er darunter den Neoliberalismus, der in Reaktion auf den Faschismus in Amerika und Deutschland eine gewaltige Konjunktur erlebte. Wenn das Grundgesetz auf die Weimarer Erfahrungen mit der Verstärkung der liberalen und der Verkürzung der sozialen Grundrechte, mit der Betonung des Föderalismus, mit einer Positionsstärkung von Kanzler und Regierung und mit der Einführung der Verfassungsgerichtsbarkeit reagierte, so wurde damit suggeriert, daß die Schwäche der Weimarer Demokratie von zuviel sozialer Demokratie, zu großer Parlamentssouveränität, vom Zentralismus und generell zu starker politischer Aktivität der Staatsbürger ausgegangen sei. Dies aber lief für Neumann auf eine Verzerrung der Krise von Weimar hinaus. ${ }^{35}$

Die politischen Imperative, die Neumann, großenteils vergeblich, wie wir wissen, an die amerikanischen Besatzungspolitik richtete, finden ihr theoretisches Pendant in den wissenschaftlichen Arbeiten nach 1950. ${ }^{36}$ Neumann war 1948 an der Columbia University zum Visiting Professor, 1950 zum Full Professor of Public Law and Government ernannt worden. In dieser Funktion verfaßte er eine Anzahl von politikwissenschaftlichen Abhandlungen, in deren Zentrum das Problem der Demokratie, ihrer Bedingungen und Möglichkeiten in der entwickelten kapitalistischen Gesellschaft steht. Er lehrte mit beachtlichem Erfolg an der Columbia University und nahm erheblichen Einfluß auf die Etablierung der politischen Wissenschaften in Deutschland, vor allem an der Freien Universität Berlin. ${ }^{37}$ Die Themen, auf die sich Neumann konzentrierte, erstreckten sich vor allem auf zwei Gebiete: auf Macht, Herrschaft, politisches System auf der einen Seite, auf die politische Philosophie, ihre Geschichte und gegenwärtige Bedeutung auf der anderen Seite. Zur Charakterisierung dieser Arbeiten muß ein methodologischer Hinweis genügen: Die Form, in der Neumann politologische Realanalyse und politische Philosophie in Beziehung setzte, deutet darauf hin, daß er eine Politikwissenschaft im Auge hatte, die weder dem Positivismus der amerikanischen political science noch dem Neonormativismus der Totalitarismustheorie verfiel. Seine Absicht war, eine »reale Theorie der Politik« im

32 Vgl. meine Einleitung zu Neumann, Wirtschaft ..., a. a. O., S. $27 \mathrm{ff}$.

33 Militärregierung und Wiederbelebung der Demokratie in Deutschland (1948), in: ebenda, S. 309 ff.

34 Deutsche Demokratie (1950), ebenda, S. 33 r.

35 Vgl. Joachim Perels, Kapitalismus und politische Demokratie, Frankfurt/M. 1973.

36 Sie sind gesammelt in: Demokratischer und autoritärer Staat, a. a. O.

37 Vgl. bes. Die Wissenschaft der Politik in der Demokratie (1950), Neumann, Wirtschaft ..., a. a. O., S. $373 \mathrm{ff}$. 
Rahmen einer vorsichtig reformulierten Gesellschaftstheorie des Spätkapitalismus zu konzipieren..$^{8}$

Erläutert sei Neumanns wissenschaftsgeschichtlicher Standort in den frühen soer Jahren anhand der Funktion, die die Verfassungs- und Rechtstheorie im Rahmen einer so verstandenen Politikwissenschaft erfüllen sollte. Zunächst bestand er, in bestimmter Negation der deutschen Tradition des Staatsrechts, darauf, daß Fragen des öffentlichen Rechts zwar in den Kanon der Politikwissenschaft aufzunehmen seien, daß sie jedoch gegenüber der sozialen Genese, der bürokratischen und verbandlichen Institutionalisierung und der Funktion von Machtprozessen in den Hintergrund zu treten haben. ${ }^{39}$ Bekanntlich reagierten beachtliche Teile der deutschen Jurisprudenz auf die Pervertierung des Rechts im Nationalsozialismus mit der sogenannten "Renaissance des Naturrechts" - eine Reaktion, die den Rechtspositivismus viel zu pauschal in den Anklagestand erhob und der sich nicht einmal so renomierter Vertreter des Relativismus wie Gustav Radbruch zu entziehen vermoch$\mathrm{te}^{40}$. Gegenüber solchen Tendenzen zu einer Rematerialisierung des positiven Rechts mußte Neumann sich abgrenzen, weil er den restaurativen Sinn des neuen Naturrechts, das sich unter modernen Bedingungen regelmäßig mit Tendenzen zur Verselbständigung der obersten Jurisprudenz gegenüber der parlamentarischen Legislative verbindet, bereits in Weimar kennengelernt und kritisiert hatte. Analog weigerte er sich jetzt, einer normativ konzipierten Theorie, und zwar auch einer solchen der Demokratie, die Königsrolle zuzugestehen, die sie in der deutschen Politik bald einzunehmen begann. So sehr er der politischen Theorie gegenüber Soziologismus und Positivismus die Aufrechterhaltung eines wahrheitstheoretischen Anspruchs abverlangte, einlösbar erschien ihm dieser nur in bestimmter Bezugnahme auf jeweilige historische und soziale Konfliktlagen und nicht im Jenseits von Geschichte und Gesellschaft. ${ }^{4}$

In ihren rechtstheoretischen Folgen ist diese Position greifbar in der großen Abhandlung "Zum Begriff der politischen Freiheit " $^{2}$. Die ausführlich abgehandelte Dimension der juristischen Freiheit erhält hier eine zwar unaufgebbare, aber doch nur negative Rolle in der modernen Gesellschaft zugesprochen, während der Akzent auf dem kognitiven Element und noch mehr auf dem Willenselement der Freiheit liegt. Diese aktiv und schöpferisch wahrzunehmen, macht allererst die Demokratie lebensfähig; ihre zunehmende Behinderung durch die Entfremdung der Bürger von der Politik, durch die Verwandlung von Kultur in Propaganda, durch die restriktive Handhabung von Grundrechten gegenüber politischen Gegnern schafft Gefahren, die eine reale Demokratie immer schwerer realisierbar machen. Diese Gefahren sah Neumann nicht nur in totalitären Gesellschaften, sondern auch in den Gesellschaften des Westens bedrohlich anwachsen, hautnah spürbar war dies etwa am loyalityProgramm der amerikanischen McCarthy-Ära. Wissenschaftsgeschichtlich signifikant ist, daß Neumann auch in seinen späten Aufsätzen, bei aller Verlagerung seines Erkenntnisinteresses, den gesellschaftstheoretischen Intentionen der materialistischen Phase treu geblieben ist - sie sind lediglich zurückhaltender formuliert und kommen über ein programmatisches Stadium nicht hinaus. Was sich also nicht bei ihm findet, sind jene von der Gesellschaftsstruktur losgelösten pluralismustheoretischen Abstraktionen, die die Politikwissenschaft der soer und 6oer Jahre alsbald zur

38 Ausführlicher dazu mein Geschichte und Herrschaft, a. a. O., S. $202 \mathrm{ff}$

39 Die Wissenschaft der Politik..., a. a. O., S. 390/39r,

40 Vgl. z. B. Radbruchs Gesetzliches Unrecht und übergesetzliches Recht (1946), und generell Wolf Rosenbaum, Naturrecht und positives Recht, Neuwied r 972.

4I Zum Begriff der politischen Freiheit (1953), in: Demokratischer und autoritärer Staat, a. a. O., S. 102 42 Ebenda. 
offiziellen Legitimationswissenschaft des westdeutschen Staates werden ließ..$^{42 a}$ Hier liegt ein wichtiger Unterschied zu seinem früheren Weggenossen Ernst Fraenkel und der von ihm begründeten sog. Berliner Schule. Interessant ist vielmehr, daß sich Neumann in seiner letzten veröffentlichten Arbeit sozialpsychologischer Methoden angenommen hat, um den bedrohlichen Zusammenhang von Angst und Politik, den er im Westen wie im Osten bedrohlich wirksam sah, zu Bewußtsein zu bringen ${ }^{43}$. Man mag darin die Richtung für einen weiteren Entwicklungsschritt sehen, den der Politikwissenschaftler zum Sozialpsychologen hätte machen können, ein Schritt, den Neumanns tragischer Tod nicht mehr zuließ.

\section{Zur Aktualität von Franz Neumann}

Theorie und Praxis des westdeutschen Rechtsstaates sind seit Anfang der 7oer Jahre erneut in Bewegung gekommen. Nach der Verunsicherung durch die Studentenbewegung und angesichts des ökonomisch erzwungenen Reformstops mobilisierte eine konservative Aufrüstung der Offentlichkeit das Legitimationsreservoir, um sicherheitsorientierte Korrekturen am rechtlich-institutionellen Gefüge der Demokratie anzubringen ${ }^{44}$. Das Resultat ist beachtlich: verfassungsgerichtlich sanktionierte Berufsverbote für Beamtenanwärter ohne entsprechendes Parteienverbot (BVGE 39, S. $334 \mathrm{ff}$.); Disziplinarverfahren gegen Beamte wegen Illoyalität (Fall Brückner); Erzwingung von Loyalitätserklärungen nicht gegenüber der Verfassung, sondern gegenüber dem Staat (Pestel gegen die Herausgeber des Mescalero-Artikels); Novellierung des Strafgesetzbuches zur Verengung der Meinungsfreiheit (StGB $\$ \$ 88 \mathrm{a}$, I 30a); Veränderung der Strafprozeßordnung und des Gerichtsverfassungsgesetzes zur Einschränkung der Verteidigerrechte, besonders das eilig verabschiedete Kontaktsperregesetz; konsequenter Ausbau des Staats-, sprich Verfassungsschutzapparats - all dies steht für wichtige, wenngleich nicht die einzigen Tendenzen der jüngsten Entwicklung ${ }^{45}$. Solche Eingriffe in den Rechtsstaat, ironischerweise als Maßnahmen zu seiner Verteidigung und Rettung durchgesetzt, sind offensichtlich alles andere als dies - sie sind bereits die ersten Schritte zu seiner Zerstörung. Franz Neumann ist aktuell, weil die mit dieser Entwicklung aufgeworfenen Fragen beantwortet werden müssen. Dazu genügt es nicht, sich jenes »rücksichtlosen Humors $\ll^{46} \mathrm{zu}$ erfreuen, der dem literarisch ambitionierten Zeitgenossen die notwendige Distanz gegenüber der tagespolitischen Realität verschafft. Grundverkehrt und hysterisch wäre es jedoch umgekehrt auch, die Bundesrepublik auf einen neuen Faschismus zutreiben zu sehen. Vielmehr bedarf es der sachlichen Besorgnis und der theoretischen Ernsthaftigkeit, d.h. der Maßstäbe, die aus dem Studium von Neumanns Biographie und Werk zu gewinnen sind wie nirgend wo anders und die aus dem theoretisch angeleiteten Blick auf die langfristigen historischen Tendenzen des deutschen Rechtsstaates resultieren.

Wir können heute erkennen, daß die Flucht der juristischen und politischen Wissenschaften in eine Renaissance des Naturrechts und damit in eine überpositive Wertordnung zum Deckmantel wird, unter dem sich problematische Tendenzen seit

42a Vgl. dazu Hans Kastendiek, Desintegration einer Integrationswissenschaft, in: Blanke, Jürgens, Kastendiek, Kritik der politischen Wissenschaft, Bd. I, Frankfurt/M. 1975, S. 27 ff.

43 Angst und Politik (1954), in: Neumann, Wirtschaft ..., a. a. O., S. $424 \mathrm{ff}$.

44 Vgl. Frankenberg u. a., Politische Tendenzwende und Entwicklung des Rechts, in: Hrsg. Tohidipur, Der bürgerliche Rechtsstaat, Frankfurt 1978, Bd. I, S. $238 \mathrm{ff}$.

4 Vgl. die Berichte des Russell-Tribunals, Berlin $1978 / 79$.

46 So Hans-Magnus Enzensberger, in: Kursbuch 56, Juni 1979, S. 14. 
der Weimarer Republik in gewandelter Gestalt fortentwickeln: die Machtsteigerung der obersten Justiz mittels des Gesetzesprüfungsrechts des Bundesverfassungsgerichts, der Überhang der öffentlichen und verbandlichen Bürokratien gegenüber den parlamentarischen Freiheitsrechten mittels des Legitimationshebels der »streitbaren Demokratie«. Solche Tendenzen mit der von Neumann analysierten Zerstörung der Rechtsrationalität im Faschismus pauschal gleichzusetzen, hieße natürlich, sich in den Maßstäben des historischen Urteils grotesk zu vergreifen. Zu fragen ist jedoch, was die von konservativen wie kritischen Beobachtern gleichermaßen konstatierten Legitimationsprobleme des gegenwärtigen Parlamentarismus in sozialer und historischer Perspektive bedeuten. Solche Legitimationsschwächen scheinen aus einem generellen Konflikt von Rechtsstaatlichkeit und rechtsstaatsüberschreitenden Steuerungsimperativen zu resultieren, ihre Lösung scheint der Mobilisierung von überpositiven Ideologien zu bedürfen.

Eine kritische Rechtswissenschaft wird an diesem Punkt anzusetzen haben. Es ist zu vermuten, daß eine theoretisch befriedigende Lösung weder in der Systemtheorie noch in der normativen Jurisprudenz, auch nicht einer kritischen, liegen kann - nicht in der Systemtheorie, weil deren "wertfreier Begriff der Legitimität « ${ }^{47}$ den Widerspruch von Recht und Macht methodisch abschneidet und Systemerhaltung selber zur scheinbar neutralen »Superlegalität " (Kirchheimer) erhebt ${ }^{48}$; nicht in der normativen Jurisprudenz, weil diese die sozialen Prozesse unterhalb der Legalitätsebene statt zu analysieren zu überdecken tendiert. ${ }^{49}$ Von Franz Neumann ist zu lernen, daß eine kritische Rechtswissenschaft drei Ebenen zu unterscheiden und wieder zusammenzukriegen hat: die Rechtsideen, die in sich verändernder Form die bürgerliche Legalität anleiten, die Rechtstheorie und Rechtspraxis selber, die unter dem Primat der Staatssouveränität steht, und das ökonomisch-soziale Substrat, unter dessen Imperativen sich Rechtsideen und Rechtsformen verändern. Eine solche Theorie, die in letzter Instanz eine historische Gesellschaftstheorie der Rechtsrationalität zu sein hätte, liegt noch nicht vor. Soll diese Aufgabe gelöst werden, so bedarf es der energischen Rezeption der Tradition, zu der Franz Neumann zusammen mit Ernst Fraenkel, Otto Kirchheimer und anderen gehört - sie darf nicht länger im Dunkel der deutschen Rechtsgeschichte verbleiben.

Alfons Söllner

47 Niklas Luhmann, Legitimation durch Verfahren, Neuwied 1969, S. 28; ders., Rechtssoziologie, Reinbek 1972 , S. $259 \mathrm{ff}$.

48 Vgl. Ingeborg Maus, Entwicklung und Funktionswandel der Theorie des bürgerlichen Rechtsstaates, in: Der bürgerliche Rechtsstaat, a. a. O., Bd. I, S. 55 ff.

49 Vgl. z. B. die Ansätze zu einer kritischen Rechtswissenschaft in: Hrsg. Jahr/Maihofer, Rechtstheorie, Frankfurt/M. 1971. 